\title{
THE MITRE MATERNAL MORTALITY INTERACTIVE DASHBOARD (3MID): A TOOL FOR ASSESSING THE EFFECTIVENESS AND EQUITY OF QUALITY IMPROVEMENT TOOLKITS ON MATERNAL CARE
}

\author{
Kevin T. Comer \\ Abdul Sheikhnureldin \\ Rachel E. Mayer \\ Model-Based Analytics \\ The MITRE Corporation \\ 7525 Colshire Drive \\ McLean, VA 22102 \\ $\{$ ktcomer, asheikhnureldin\}@mitre.org \\ CVET + Strategy, Government, and Acquisition \\ The MITRE Corporation \\ 7525 Colshire Drive \\ McLean, VA 22102 \\ remayer@mitre.org
}

\author{
Sybil A. Klaus \\ Health Transformation Tech Center \\ The MITRE Corporation \\ 7525 Colshire Drive \\ McLean, VA 22102 \\ sklaus@mitre.org
}

\begin{abstract}
From 1987 to 2014, the U.S. pregnancy-related mortality rate increased from 7.2 deaths per 100,000 live births to 18 deaths per 100,000 live births (Centers for Disease Control and Prevention, 2018). Of the pregnancy-related deaths in the United States, $66 \%$ are preventable (Petersen, et al., 2019). We identified key factors that influence maternal mortality rates and incorporated related data into an interactive dashboard and policy microsimulation entitled the MITRE Maternal Mortality Interactive Dashboard (3MID). This analytic tool, built in R/Shiny, allows us to visualize historic trends at the state level and build a microsimulation to forecast future trends in maternal mortality, docking the results of the simulation to empirical data from the Centers for Disease Control and Prevention. Finally, we can explore the range of expected outputs of various policies aimed at ameliorating the causes of pregnancy related deaths, particularly among non-Hispanic black mothers in various states.
\end{abstract}

Keywords: maternal health, healthcare policy, microsimulation

\section{INTRODUCTION}

The pregnancy-related mortality rate in the United States has doubled in the last two decades, leading to a crisis in women's health (Morton, VanOtterloo, Seacrist, \& Main, 2019). From 1987 to 2014, the U.S. pregnancy-related mortality rate increased from 7.2 deaths per 100,000 live births to 18 deaths per 100,000 live births (Centers for Disease Control and Prevention, 2018). Importantly, non-Hispanic black women are more than three to four times more likely to die from a pregnancy-related death (Howell, 2018). 
This health crisis has prompted many states to stand up research committees and partnerships for the purposes of reducing maternal morbidity and mortality. One of the most successful of these committees is the California Maternal Quality Care Collaborative (CMQCC), which has published a number of "toolkits" for the purposes of addressing the most common causes of preventable pregnancy-related deaths. Each toolkit, focused on a single cause of mortality or morbidity, includes a "compendium of best practice tools and articles, care guidelines in multiple formats, hospital-level implementation guide, and professional education slide set." (CMQCC: California Maternal Quality Care Collaborative, 2019). The toolkits currently in circulation are listed in Table 1, focused on the most prevalent of the causes of pregnancy related mortality (Creanga, Syverson, Seed, \& Callaghan, 2017).

Table 1: Toolkits Developed by the CMQCC.

\begin{tabular}{|l|l|}
\hline Quality Improvement Toolkit & Maternal Deaths Per 100,000 Live Births \\
\hline $\begin{array}{l}\text { Improving Health Care Response to Cardiovascular } \\
\text { Disease in Pregnancy and Postpartum }\end{array}$ & 4.42 \\
\hline Improving Health Care Response to Preeclampsia & 1.25 \\
\hline $\begin{array}{l}\text { Improving Health Care Response to Maternal Venous } \\
\text { Thromboembolism }\end{array}$ & 1.56 \\
\hline Improving Response to Obstetric Hemorrhage & 1.93 \\
\hline Support Vaginal Birth \& Reduce Primary Cesareans & $\begin{array}{l}5.80 \text { - by direct cause of caesarean delivery } \\
(\text { Sachs 1989) }\end{array}$ \\
\hline Elimination of Elective Deliveries Before 39 Weeks & $\begin{array}{l}\text { N/A - aimed at reducing risk of other risk factors } \\
\text { of mortality (preeclampsia, etc.) (CMQCC: } \\
\text { California Maternal Quality Care Collaborative } \\
\text { 2011) }\end{array}$ \\
\hline
\end{tabular}

With the development of these toolkits beginning in 2006, and their implementation in 2009 (Main, Markow, \& Gould, 2018), the rate of pregnancy-related deaths in California by 2013 has been cut in half, to a three-year average of 7.0 deaths per 100,000 live births. However, while the success of these toolkits has been seen across the state's population, the disparity between the mortality rate of non-Hispanic white and black women has remained relatively constant - as of 2013, non-Hispanic black women have a 3.4 times higher mortality ratio than non-Hispanic white women (Creanga, Syverson, Seed, \& Callaghan, 2017). The most prevalent pregnancy-related causes of death are shown in Table 2, as noted in the Maternal Mortality Review Information Application (MMRIA) report from 2018 (Brantley, et al., 2018):

Table 2: Leading Causes of Pregnancy-Related Deaths in the United States.

\begin{tabular}{|l|l|l|l|}
\hline Cause of Death & $\begin{array}{l}\text { Total } \\
\text { Percent } \\
\text { of Deaths }\end{array}$ & $\begin{array}{l}\text { Percent of } \\
\text { Non-Hispanic } \\
\text { White Deaths }\end{array}$ & $\begin{array}{l}\text { Percent of } \\
\text { Non-Hispanic } \\
\text { Black Deaths }\end{array}$ \\
\hline Cardiovascular and coronary conditions & $14.0 \%$ & $15.5 \%$ & $10.5 \%$ \\
\hline Hemorrhage & $14.0 \%$ & $14.4 \%$ & $12.8 \%$ \\
\hline Infection & $10.7 \%$ & $13.4 \%$ & $8.1 \%$ \\
\hline Cardiomyopathy & $10.7 \%$ & $10.3 \%$ & $14.0 \%$ \\
\hline Preeclampsia and eclampsia & $8.4 \%$ & $5.2 \%$ & $11.6 \%$ \\
\hline
\end{tabular}




\begin{tabular}{|l|l|l|l|}
\hline Embolism & $7.4 \%$ & $5.2 \%$ & $9.3 \%$ \\
\hline
\end{tabular}

While California has seen great success in reducing their rates of pregnancy-related mortality with the implementation of quality improvement toolkits, other states and stakeholders are challenged with estimating the impact they would receive by utilizing similar toolkits. This paper discusses the microsimulation, developed by the paper's authors, to simulate a population of pregnant women in a given state within the United States. Using demographic data from the U.S. Census Bureau, as well as data from the CMQCC and other similar collaboratives across the country, this model simulates the effect of certain toolkits, as listed in Table 1, on a synthetic population. Using this quantitative analysis, users can assess which toolkits would create the most impact to the reduction of maternal mortality, as well as the BlackWhite maternal mortality disparity rate..

\section{DATA SOURCES}

The microsimulation described in this paper, known as the MITRE Maternal Mortality Interactive Dashboard (3MID) operates off of a synthetic population of pregnant women, used to represent the population of actual women within the locality being simulated, usually a U.S. state such as California. The reason for using a synthetic population, rather than a real population, is the ability to utilize realistic data in a simulation run and witness the impacts of these experiments without the concern for privacy as ensured in the Health Insurance Portability and Accountability Act of 1996 (HIPAA). The data used to construct this synthetic population was drawn from the U.S. Census Bureau American Community Survey, which captures a number of attributes of the American population such as income, employment, locality, number of individuals in a household, age, and race/ethnicity.

A synthetic population is a reproduced representation of a population which replicates the statistical variable distributions of a population. With the use of Synthetic Populations and Ecosystems of the World (SPEW) (Gallagher, Richardson, Ventura, \& Eddy, 2018), the 3MID tool is able to construct a population with demographic and geographical distributions that are currently being docked to distributions defining the actual population, as captured from the American Community Survey from the U.S. Census Bureau. As with many other microsimulations focused on the health of specific subpopulations, such as with unwanted pregnancies (Karpilow, Cook, \& Manlove, 2017) or the effects of treatment on breast cancer (Birnbaum, Gadi, Markowitz, \& Etzioni, 2016), this microsimulation will be modelling on women of child-bearing potential, ages 14 through 44 . For the purposes of attributing causes of pregnancy-related mortality among women, the tool utilized data drawn from the Healthcare Cost and Utilization Project (HCUP), Institute for Health Metrics and Evaluation (IHME), and March of Dimes PeriStats.

3MID primarily focuses on the white non-Hispanic and black non-Hispanic populations, including causes and rates of maternal mortality. This is due to the acknowledged disparity between these two populations with regards to death rates and overall maternal health, as well as the overall high number of cases that can inform the model, due to larger populations in California and across the country. Given the success of this methodology, 3MID could be extended to address other populations of women of different ethnicities, such as Hispanic, Native American, Pacific Islander, etc. (Petersen, et al., 2019).

\section{THE MITRE MATERNAL MORTALITY INTERACTIVE DASHBOARD (3MID)}

The tool was developed using R and the Shiny framework (Chang, Cheng, Allaire, Xie, \& McPherson, 2019) to combine both advanced data analytics capabilities with rapid and interactive visualization techniques. This approach allows for computationally efficient calculations in the back end with a visually pleasing front end incorporating ggplot2, ploypy, and JavaScript libraries leveraged by Shiny.

After the 3MID tool has constructed the synthetic population of pregnant women for a state from the drop down menu, the model simulates the impact of the various causes of pregnancy-related mortality might 
have on that population, as listed in Table 2. The individual entities (i.e., synthetic pregnant women) are then mapped across the state in question, with counties color coded according to aggregate risk values, and entities color coded according to race (blue for non-Hispanic white, green for non-Hispanic black). A sample display is shown in Figure 1 below.

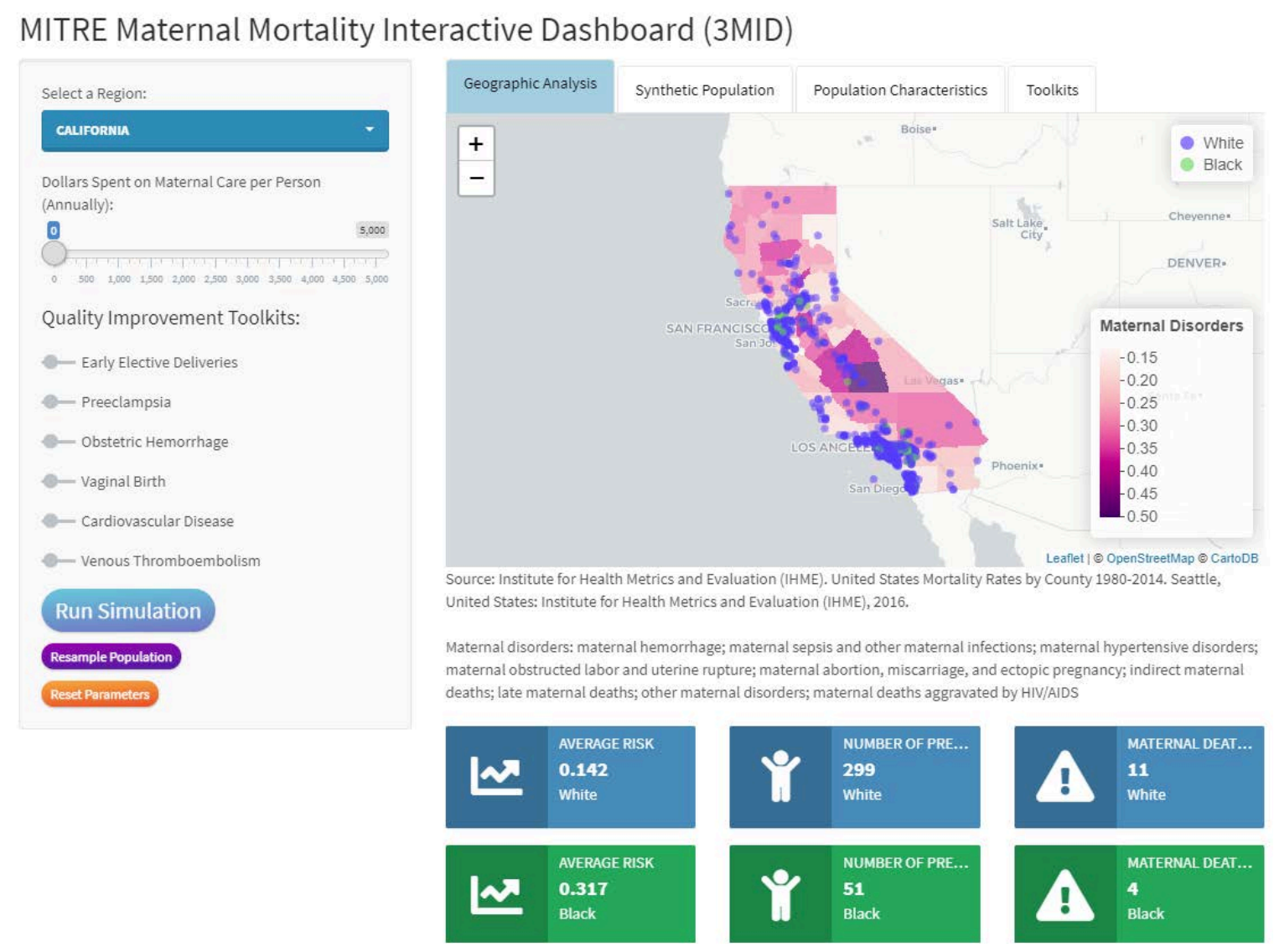

Figure 1: Screenshot of a Single Run of the 3MID Tool.

Hovering over each dot in the geographical window shows the information generated for that synthetic individual, including race, income, employment status, and most notably, the risk of mortality to a pregnancy-related cause. Below the geographical window, the various measures of effectiveness are listed, including the average risk to Non-Hispanic White and Black Women, and the Number of PregnancyRelated Deaths. The risk of mortality is currently calculated as a linear regression model of the individual's race, age, and county of residence. These results are based on mortality data derived from high level reports from the Agency for Healthcare Research and Quality (AHRQ) that are based on the HCUP data.

$$
\rho_{i}=\beta_{0}+\beta_{1} x_{1}+\beta_{2} x_{2}+\beta_{3} x_{3}+\varepsilon,
$$

where $x_{1}$ is the individual's age, $x_{2}$ represents the individual's race ( 0 for non-Hispanic white, 1 for nonHispanic Black), and $\mathrm{x}_{3}$ is the aggregate county risk of mortality. Validation of the aggregate county risk variable as a function of income and education of the residents is being performed. On account of the low number of observations in the empirical data $(\mathrm{N}<25$ in most counties each year), validation of the model will be performed using t-tests, comparing the results of the model with the empirical data collected on maternal mortality. Once the base rate of mortality is determined, the mortality by cause is defined for each individual entity, using the probabilities of various possible causes of pregnancy-related causes of death, as described in Table 2. 


$$
\rho_{i j}=\frac{r_{j 0}\left(1-x_{2}\right)+r_{j 1} x_{2}}{r_{j}},
$$

where $\mathrm{j}$ is the specific cause of mortality (Hemorrhage, infection, etc.), $\mathrm{r}_{\mathrm{j}}$ is the base rate of mortality for cause of mortality across the population, and $r_{j 0}$ and $r_{j 1}$ are the rates of mortality by cause $j$ for non-Hispanic white women and black women respectively ( $\mathrm{x}_{2}$ represents the individual's race, as above).

After establishing a baseline scenario, wherein the synthetic population has been generated and individual risks of mortality have been calculated, various toolkits from Table 1 can be simulated, and their effects on the population be assessed. The user can select both the toolkits to be enacted, and the level of funding to be provided to the implementation of those toolkits. Since implementation of the toolkits is not universal to the population, a level of funding for the toolkit brings a common stakeholder decision into play by determining how much the toolkit can impact the health of the population for a given cause of pregnancyrelated mortality. The effectiveness of each toolkit to reduce the rate of death for each cause is determined as an input by the level of funding provided. Once the toolkit(s) in question have been selected, the simulation can be rerun on the same population. To ensure a further degree of randomness, a new population can be generated for the purposes of maintaining the robustness of the policy simulation by understanding the change in the output variables as a direct result of the implementation of the toolkits.

Each individual risk of pregnancy-related mortality is evaluated independently, depending on which toolkits are being simulated in a given run. The toolkits are effective only against the causes of mortality they are aimed to prevent, so for instance the Improving Health Care Response to Preeclampsia toolkit will not prevent a death caused by infection. This is despite there being evidence that there are comorbidities that do cross several causes of death, which have not been included into the model. This is based on an assumption of the 3MID developers that higher levels of funding to maternal care facilities, for the purposes of training, technology, and additional qualified personnel, by state will lead to reduced pregnancy-related deaths.

\section{CONCLUSION}

To combat the increasing prevalence of preventable maternal mortality in the United States, especially among Non-Hispanic Black women, the authors have developed the MITRE Maternal Mortality Interactive Dashboard (3MID). 3MID is an interactive dashboard and microsimulation to be used for the analysis of the impact of policy on disparate populations. This analytic tool, built in R/Shiny, allows us to build a forecast model to predict future trends in maternal mortality. The microsimulation establishes a baseline synthetic population from R/SPEW, based on demographic data drawn from the U.S. Census and the American Community Survey. We incorporate the six leading causes of pregnancy-related mortality into 3MID and dock the results of the simulation to empirical data from the Centers for Disease Control and Prevention, where available from 2002 to 2017. This impact can be shown both statistically, as the results of Monte Carlo simulation, and graphically on highly detailed state maps built in $\mathrm{R}$ and shown via Leaflet, which are populated by both county-level maternal mortality and hospital data. The goal is for 3MID to be used by policymakers to allocate resources and optimize the impact of limited resources on maternal mortality and racial disparities within maternal health.

Tool development is still needed in validating and quantifying the assumption referred to in Section 3 namely, the extent that funding towards various quality improvement toolkits increases the per capita efficacy of the toolkit at reducing the cause of pregnancy-related mortality. Similarly, the impact of comorbidities on rates of pregnancy-related causes of death, while known, have not yet been quantified in $3 \mathrm{MID}$, and so more development will be needed in coding these elements into the synthetic population. 


\section{ACKNOWLEDGMENTS}

The authors' affiliation with The MITRE Corporation is provided for identification purposes only, and is not intended to convey or imply MITRE's concurrence with, or support for, the positions, opinions, or viewpoints expressed by the author. Approved for Public Release; Distribution Unlimited. Public Release Case Number 19-3766.

\section{REFERENCES}

Birnbaum, J., Gadi, V. K., Markowitz, E., \& Etzioni, R. (2016). The Effect of Treatment Advances on the Mortality Results of Breast Cancer Screening Trials: A Microsimulation Model. Annals of Internal Medicine, 236-243.

Brantley, M. D., Callaghan, W., Cornell, A., Cox, S., Davis, N., Foster, S., . . Zaharatos, J. (2018). Report From Nine Maternal Mortality Review Committees.

Centers for Disease Control and Prevention. (2018). Pregnancy Mortality Surveillance System. Retrieved from https://www.cdc.gov/reproductivehealth/maternal-mortality/pregnancy-mortalitysurveillance-system.htm

Chang, W., Cheng, J., Allaire, J., Xie, Y., \& McPherson, J. (2019, October 10). shiny: Web Application Framework for R. R package version 1.4.0.

CMQCC: California Maternal Quality Care Collaborative. (2011). Elimination of Non-medically Indicated (Elective) Deliveries Before 39 Weeks Gestational Age, A California Toolkit to Transform Maternity Care. Stanford, CA.

CMQCC: California Maternal Quality Care Collaborative. (2019). Toolkits. Retrieved from https://www.cmqcc.org/resources-tool-kits/toolkits

Creanga, A. A., Syverson, C., Seed, K., \& Callaghan, W. M. (2017). Pregnancy-Related Mortality in the United States, 2011-2013. Obstetrics and Gynecology, 366-373.

Gallagher, S., Richardson, L. F., Ventura, S. L., \& Eddy, W. F. (2018). SPEW: Synthetic Populations and Ecosystems of the World. Journal of Computational and Graphical Statistics, 773-784.

Howell, E. A. (2018). Reducing Disparities in Severe Maternal Morbidity and Mortality. Clinical Obstetrics and Gynecology, 387-399.

ICD10data.com. (2019). $\quad$ ICD-10 Codes. $\quad$ Retrieved 2019, $\quad$ from https://www.icd10data.com/ICD10CM/Codes

Karpilow, Q., Cook, E. L., \& Manlove, J. (2017). A microsimulation model of fertility, childbearing, and child well-being. International Journal of Microsimulation, 3-58.

Main, E. K., Markow, C., \& Gould, J. (2018). Addressing maternal mortality and morbidity in California through public-private partnerships. Health Affairs, 37(9), 1484-1493.

Morton, C. H., VanOtterloo, L. R., Seacrist, M. J., \& Main, E. K. (2019). Translating Maternal Mortality Review Into Quality Improvement Opportunities in Response to Pregnancy-Related Deaths in California. JOGNN - Journal of Obstetric, Gynecologic, and Neonatal Nursing, 252-262.

Petersen, E. E., Davis, N. L., Goodman, D., Cox, S., Mayes, N., Johnston, E., . . Barfield, W. (2019). Vital signs: Pregnancy-related deaths, united states, 2011-2015, and strategies for prevention, 13 states, 2013-2017. Morbidity and Mortality Weekly Report, 423-429. 
Petersen, E. E., Davis, N. L., Goodman, D., Cox, S., Syverson, C., Seed, K., . . . Barfield, W. (2019). Racial/Ethnic Disparities in Pregnancy-Related Deaths - United States, 2007-2016. Washington, D.C.: US Department of Health and Human Services/Centers for Disease Control and Prevention.

Sachs, B. P. (1989). Is the Rising Rage of Cesarean Sections a Result of More Defensive Medicine? In I. o. Care, Medical Professional Liability and the Delivery of Obstetrical Care: Volume II: An Interdisciplinary Review. Washington, D.C.: National Academies Press.

\section{AUTHOR BIOGRAPHIES}

KEVIN T. COMER is a Senior Simulation Modeling Engineer at The MITRE Corporation. He received a Ph.D. from George Mason University in Computational Social Science in 2017, focusing on the simulation of healthcare policy. He is currently an adjunct professor at George Mason University teaching courses in Economics and Computational Social Science, and can be contacted at ktcomer@mitre.org.

ABDUL SHEIKHNURELDIN is a Data Scientist in the Model-Based Analytics department at The MITRE Corporation. As a member of the Computational Social Science group, he is the primary developer of the MITRE Maternal Mortality Interactive Dashboard. He received his BA from The George Washington University, where he double majored in Computer Science and Political Science. His email is asheikhnureldin@mitre.org

RACHEL E. MAYER is a Senior Health Systems Analyst and Principal Investigator at the MITRE Maternal Mortality Interactive Dashboard Project through The MITRE Innovation Program. Her research interests include maternal and child health, Veteran health, and social and behavioral science. She received her MPH from LSU Health Sciences Center in New Orleans and a BA in psychology from the University of Miami. Her email is remayer@mitre.org.

DR. SYBIL A. KLAUS is the Chief Scientist for the Health Transformation Tech Center at the MITRE Corporation. She is The MITRE Innovation Program's Health Innovation Area Leader and works on provider data optimization. Sybil is a trained pediatrician Sybil obtained dual degrees in political science and cell and molecular biology followed by medical degree with an internship and residency in Pediatrics, and a Master of Public Health. Her email is sklaus@mitre.org. 